\title{
Diseño de un marteloscopio para la simulación de gestión selvícola de alcornocal en condiciones reales
}

\section{Design of marteloscope for simulation of silvicultural management of cork oak forest under field conditions}

\author{
Begoña Abellanas Oar*, Fernando Baldero Echarren, Luis Guada González, \\ Simón Cuadros Tavira, Pedro J. Pérez Moreno, Álvaro Sellez Ruiz, Enrique \\ Urbano Fuentes-Guerra
}

Fecha de recepción: 18/10/2019; Fecha de revisión: 23/01/2020; Fecha de aceptación: 12/02/2020

Cómo citar este artículo:

Abellanas,B., Baldero, F., Guada, L., Cuadros, S., Pérez, P.J., Sellez, A. \& Urbano, E. (2020). Diseño de un marteloscopio para la simulación de gestión selvícola de alcornocal en condiciones reales. Revista de Innovación y Buenas Prácticas Docentes, 9(1), 1-12.

Autor de Correspondencia: ir1aboab@uco.es

\begin{abstract}
Resumen:
Un marteloscopio es una herramienta didáctica de gran utilidad para el entrenamiento en selvicultura. La disponibilidad de datos reales sobre la masa forestal en dispositivos móviles (tablets) en combinación con un software adecuado permite la retroalimentación directa entre las decisiones selvícolas y los resultados, tanto económicos como ecológicos de las mismas. A través de este Proyecto de Innovación Docente, se ha avanzado en esta línea formativa diseñando e implementando, por primera vez, un marteloscopio en monte alcornocal orientado a la producción de corcho. Como resultado, se ha establecido una parcela permanente de entrenamiento en un alcornocal, que se ha monitorizado de forma exhaustiva, y se ha desarrollado un software nuevo para $p c$ o tablet que simula en tiempo real los efectos de ejercicios de claras selvícolas y de descorches, que el usuario introduce, y que genera salidas instantáneas sobre la producción de corcho obtenida y sus características y sus efectos sobre la masa forestal. Esta simulación facilita el aprendizaje realista de la gestión de alcornocales y la comprensión de los efectos que las decisiones de gestión tomadas tienen sobre los aspectos productivos y ecológicos de la masa forestal.
\end{abstract}

Palabras clave: Biodiversidad, Gestión forestal, Juego de simulación, Producción de corcho, Selvicultura

\begin{abstract}
:
A Marteloscope is an educational tool for silvicultural and forest management training. The availability of actual stand data on mobile devices (tablets) in combination with the appropriate software allows direct feedback among silvicultural decisions and the resulting economic and ecological outcomes. Through this Teaching Innovation Project, we have gone further on this approach by designing and implementing, for the first time, a Marteloscope in a cork oak stand managed for cork production. As a result, we have set up a permanent training plot in the forest, where all trees have been geolocated, measured, both in size and cork production features, marked and also assessed for their ecological value through microhabitat identification approach. We have developed new software for $p c$ or tablets to simulate in real-time silvicultural and cork stripping exercises made by the user, producing instantaneous outputs showing the effects on cork production and forest stand, both currently and after 9 years period (duration of debarking rotation). This simulation facilitates realistic learning of cork oak stands management practices and the understanding of the effects of management decisions on productive and ecological dynamics of forest stand.
\end{abstract}

Key Words: Biodiversity, Cork production, Forest management, Silviculture, Simulation game 


\section{INTRODUCCIÓN}

Este proyecto da continuidad a la labor iniciada en un proyecto precedente orientado a trasladar los conceptos teóricos a la práctica en un campo como el forestal de difícil consecución dentro de las infraestructuras de un Campus Universitario (Abellanas et al., 2018). La toma de decisiones de gran parte de las actuaciones forestales conducentes a la gestión y el aprovechamiento de los montes deben tomarse, en gran medida, sobre el terreno.

La ausencia de campos de experimentación adecuados en este ámbito hace necesario que las enseñanzas teóricas y prácticas en clase se complementen con actividades dirigidas sobre el terreno. Esto requeriría disponer de una red adecuada de parcelas de entrenamiento dentro del propio hábitat forestal. Por otro lado, las actuaciones forestales no suelen producir los efectos esperados de forma inmediata, sino tras dilatados procesos de respuesta por parte del sistema forestal, cuya dinámica es extremadamente lenta, lo que complica el aprendizaje de los efectos que las actuaciones selvícolas ejercen a largo plazo.

Por todo ello, entendemos que es muy necesario completar el aprendizaje en aula con un trabajo en campo que combine la toma de decisiones por parte del alumno, supervisada por el profesorado, con la simulación en tiempo real del efecto de las actuaciones propuestas para que los alumnos puedan entender el alcance de las decisiones de gestión adoptadas, tanto en relación a los aprovechamientos que se pretendan obtener con tales actuaciones como sobre el componente ecológico que, en todo caso, debe preservarse en cualquier actuación forestal.

En el ámbito mediterráneo existen aprovechamientos forestales de gran valor como el corcho, las setas o los frutos forestales que no se obtienen al final de la vida de los árboles, como la madera, sino durante la vida de la masa forestal. Esto obliga a introducir premisas específicas dentro del manejo forestal en relación con la gestión forestal tradicional de corte centroeuropeo, donde el producto principal -la madera- se obtiene al final de la vida de los árboles. Especialmente singular es el aprovechamiento del corcho, uno de los productos directos más valiosos actualmente en el monte mediterráneo. Los sucesivos descorches a lo largo de la vida de los árboles pueden influir decisivamente en las producciones futuras y en el estado de la masa forestal, por lo que se hace necesario incorporar modelos de futuro que permitan optimizar los aprovechamientos a distintas escalas temporales. La combinación del diseño selvícola óptimo para conformar y gestionar el monte alcornocal con las prescripciones adecuadas para la realización de los sucesivos aprovechamientos del corcho es un proceso de decisión complejo que se entenderá mejor si se trabaja con casos reales y con modelos predictivos que permitan simular las consecuencias a corto, medio y largo plazo de las actuaciones programadas simultáneamente a la toma de decisiones.

El interés del presente proyecto ha sido preparar un dispositivo que aúna la selección de un escenario controlado en campo en monte alcornocal con la puesta a punto de un modelo de simulación que puede emplearse de forma ágil sobre el terreno, como por ejemplo en una tablet o smartphone, con el fin de proporcionar una herramienta de entrenamiento en campo para los alumnos de diversas asignaturas de las titulaciones de Grado en Ingeniería Forestal y Máster en Ingeniería de Montes, entre ellas: Selvicultura Mediterránea, Aprovechamientos Forestales, Dasometría e Inventariación Forestal, Ordenación de Montes, Gestión de Espacios naturales protegidos, y otras. 


\section{DESARROLLO DE LA EXPERIENCIA DE INNOVACIÓN}

\subsection{Objetivos}

El proyecto desarrollado se ha planteado tres de los principales objetivos recogidos en el Plan de Innovación y Buenas prácticas Docentes 2017-2018 de la UCO:

1. Desarrollar una acción de innovación docente que genere una mejora de los procesos de enseñanza-aprendizaje y de los resultados académicos del alumnado.

2. Diseñar una práctica docente innovadora y que pueda constituir un referente de calidad, incorporando una metodología novedosa consistente en la combinación de trabajo real en campo con simulación virtual en tiempo real del efecto futuro de las actividades planteadas sobre el terreno.

3. Poner en práctica una metodología docente centrada en la actividad del alumnado que permita a éste fijar los conceptos teóricos aprendidos en clase y trasladarlos a su aplicación efectiva en la simulación de algunas de las tareas que constituirán parte de su futura labor profesional.

Como objetivos concretos se han planteado los siguientes:

a) Establecer una parcela de entrenamiento en monte alcornocal en la Finca "Pino Gordo", situada en la Sierra de Córdoba, para la ejecución de actividades universitarias dirigidas in situ y la simulación de un campo de experimentación adecuado con base en tecnologías informáticas que complemente el aprendizaje de alumnos a partir de habilidades académicas como la toma de decisiones y como la simulación de tratamientos selvícolas y de aprovechamientos de corcho.

b) Desarrollar la base del software de trabajo que se utilizará en campo en las simulaciones de los alumnos basado en la adaptación de modelos simplificados de crecimiento y producción de corcho existentes en la literatura, así como en el cálculo del valor económico y ecológico que resulte de las actuaciones simuladas.

c) Aplicar el método en prácticas tutoradas con los alumnos y analizar el funcionamiento de este a través de la evaluación del aprendizaje y competencias adquiridas.

\subsection{Material y métodos}

De acuerdo con los objetivos planteados en el apartado anterior, se han desarrollado las siguientes tareas y actividades:

a) Elección y establecimiento de la parcela

La parcela de experimentación en monte alcornocal se ha establecido en la Finca "Pino Gordo" situada en la Sierra de Córdoba (T.M. Córdoba) y donde el alcornocal constituye el sistema forestal dominante. Se trata de una finca particular, siendo el gestor de la misma Colaborador Honorario del Departamento de Ingeniería Forestal y miembro integrante del Equipo propuesto para este proyecto de Innovación Docente, lo que ha facilitado la realización de los trabajos y su integración en la formación práctica de los alumnos de la UCO. La parcela tiene forma rectangular y una superficie de 0,6 ha en proyección horizontal. Esta parcela se ha monitorizado de 
forma exhaustiva mediante la realización de un mapa de árboles y la inventariación detallada de los mismos mediante la utilización del dispositivo Field-Map, con registro de: número de identificación, coordenadas, proyección de copas, especie, medidas dendrométricas como: altura total, $\mathrm{H}$, altura de fuste, $\mathrm{Hf}$, Circunferencia normal, CSC, espesor de corteza, EC, superficie de proyección de la copa, SC, y, en el caso de alcornoques, medidas ligadas a la producción corchera como: altura de descorche, $H D$, y sus variaciones en las últimas pelas, superficie de descorche, SD, etc. También se ha hecho un reconocimiento exhaustivo de la presencia de microhábitats relevantes para albergar biodiversidad en cada uno de los árboles de la parcela, de acuerdo con el catálogo propuesto por Kraus et al. (2016). Estos registros han conformado la base de datos con la que se ha construido el modelo de simulación y su implementación en una herramienta Excel.

La simulación pretendida requiere que los usuarios puedan identificar fácilmente cada uno de los árboles de la parcela, por lo que ha sido necesario marcar los mismos con un código numérico que resultara visible a distancia. Esto se ha realizado con pintura ecológica específica para el marcado de árboles y la utilización de plantillas numéricas (Figura 1).


Figura 1. Mapa de árboles y proyección de las copas de los árboles obtenido con el dispositivo Field Map (izqda.) y aspecto de la parcela durante su levantamiento (dcha.)

\section{b) Elaboración de la herramienta informática para los usuarios}

Se ha desarrollado una herramienta informática de uso sencillo que permite simular actuaciones selvícolas y de aprovechamiento de corcho por los usuarios (alumnos) así como la obtención de los resultados de las mismas, tanto en términos económicos (producción de corcho) como en términos ecológicos, estimados estos últimos a través de determinación del valor ecológico del sistema basado en la biodiversidad del mismo. La simulación incorpora no sólo los resultados inmediatos de la práctica ejecutada sino también una simulación realista de los efectos futuros de las actuaciones propuestas sobre dichos parámetros, habida cuenta que tanto el aprovechamiento del corcho como las medidas selvícolas que se planteen 
(fundamentalmente claras) tendrán efectos tanto sobre los futuros aprovechamientos como sobre la dinámica de la masa forestal.

Como medidas relacionadas con el descorche se trabaja con las siguientes, de acuerdo con la norma habitual:

Superficie de descorche (SD) del árbol, calculada según la norma como el producto entre la Circunferencia normal bajo corcho (CBC) y la altura de descorche (HD). No se contempla el descorche de ramas, que actualmente tiende a abandonarse por las desventajas que presenta, tanto en rendimientos y calidad del corcho como en el crecimiento y vigor de los árboles. En todo caso, en la parcela no hay ningún alcornoque descorchado en ramas y el simulador no contempla esta opción.

Intensidad de descorche (ID) del árbol, calculada como el cociente entre la superficie de descorche (SD) y la sección normal (a 1,30 m) del árbol bajo corcho, $g$.

La herramienta informática (en base Excel) permite obtener una caracterización actual de la parcela, tanto en términos dasométricos como ecológicos y productivos, con salidas gráficas (funciones de densidad y mapas de árboles con indicación espacial de distintas variables dendrométricas, ecológicas y productivas de la población de árboles de la parcela) y numéricas (variables dasométricas, ecológicas y productivas de la masa). También se ha configurado una salida correspondiente a la simulación de la evolución de la parcela a los 9 años tras los tratamientos ensayados en la actualidad. En este caso, se utiliza el modelo de crecimiento que se describe a continuación con todos los árboles de la parcela (si no se simula clara) o sólo con los árboles remanentes si se simula una clara en el momento actual. En esta proyección a 9 años se obtienen los valores ligados a la producción de corcho mediante una simulación de descorche estándar (no intervenida por el usuario) con las mismas alturas de descorche de partida (se simula un descorche con las alturas de descorche medidas en monte, que se corresponden con el último descorche real ejecutado).

Pero su utilidad más interesante es la simulación de actuaciones: tanto de aprovechamiento del corcho como de gestión selvícola.

Las actuaciones que se puedes simular son:

. Respecto al aprovechamiento de corcho, las decisiones de gestión básicas a tomar y que pueden simularse son: la determinación del tamaño mínimo de los árboles para iniciar el descorche (desbornizamiento) y la altura de descorche a aplicar a cada árbol. Ambas variables pueden simularse en el sistema con las siguientes restricciones:

La Circunferencia sobre corcho (CSC) mínima para el desbornizamiento está limitada a un valor mínimo de $65 \mathrm{~cm}$, que es lo que marca la normativa actual. Pero el gestor (usuario) puede decidir aumentar dicho valor mínimo. Es una restricción genérica para todos los árboles de la parcela.

La altura de descorche (HD) está limitada a la altura de fuste del árbol pues no se contempla el descorche de ramas. Puesto que la parcela se encuentra ya en producción, gran parte de los arboles presentan ya una altura previa de descorche. Sobre dicha altura, el gestor puede decidir aumentarla o disminuirla. Es una restricción aplicable a cada árbol, ya que depende de su propia altura de fuste. 
El sistema está pensado para simular tratamientos y aprovechamientos en el momento actual, aun cuando la masa se ha medido a la mitad del turno de descorche. Lo relevante del sistema no es obtener valores reales de producción sino estudiar sobre el terreno y con valores realistas las implicaciones de las actuaciones sobre la respuesta en producción y en características dasométricas y ecológicas de la masa resultante y su evolución en el tiempo.

. Respecto a la gestión selvícola de la masa, la decisión a tomar, y que puede simularse, es la realización de claras. Se contempla también la posibilidad de marcar árboles de porvenir.

Las simulaciones de claras y aprovechamientos se pueden hacer conjuntamente o por separado. Como resultados se obtienen dos opciones:

i) el resultado inmediato:

- De la simulación de una clara se obtiene como resultado la variación de la masa resultante en características dasométricas, ecológicas y productivas.

- De la simulación del aprovechamiento de corcho se obtiene como resultado: la producción de corcho obtenida y su variación respecto al último descorche realizado (En superficie de descorche, que puede transformarse en peso aplicando el valor medio de la densidad superficial del corcho, PCM2, del monte y éste en valor económico aplicando los precios medios del corcho por unidad de peso), distinguiendo entre corcho de reproducción y bornizo. La variación de la intensidad de descorche es también un resultado de interés que se obtiene, tanto para el total de la masa como por clases diamétricas e incluso por árbol individual.

Si se simulan ambas cosas simultáneamente, se supone que el aprovechamiento es previo a la clara, por lo que la producción de corcho se obtiene del conjunto de la masa inicial, aun cuando el sistema permite diferenciar la parte obtenida de la masa residual y aquella obtenida de la masa cortada.

ii) el resultado al cabo de 9 años:

la influencia de las actuaciones sobre la producción y estado de la masa al cabo de 9 años (siguiente turno simulado de descorche)

Para esto, se ha utilizado una modificación del modelo de crecimiento CORKFITS (Ribeiro y Surovy, 2011) que permite simular el crecimiento en grosor (de la sección normal, $g$ ) de los árboles (Incg) en función de su tamaño inicial (sección normal bajo corcho, $g$ ), del grado de competencia al que está sometido por sus vecinos (a través del índice de competencia de Hegyi, que tiene en cuenta la relación entre la distancia a los vecinos y su relación de tamaños) y de la intensidad de descorche a que ha sido sometido, ID:

\section{Incg=IncgPot ${ }^{\star}$ modificador}

Incgpot $=f(g)$, es el crecimiento potencial en sección normal de un árbol que no presente competencia de otros árboles ni haya sido descorchado y depende exclusivamente del tamaño inicial del árbol.

modificador= $f($ Hegyi, ID), es el modificador (reductor) del crecimiento del árbol debido a la competencia de los vecinos (Indice de competencia de Hegyi) y a la 
Intensidad de descorche sufrida por el árbol (ID), de forma que el crecimiento del árbol se verá tanto más reducido respecto a su crecimiento potencial, cuanto mayor sea la competencia ejercida por los vecinos (que se puede hacer variar a través de la simulación de claras) y cuanto mayor sea la intensidad del descorche al que se someta al árbol (ID), que también se puede variar en la simulación, dentro de las restricciones especificadas.

Mediante este simulador del crecimiento, las actuaciones realizadas, además de afectar al número de árboles (en el caso de la clara), y a la cantidad de corcho extraído en la simulación del descorche actual, afectan también al crecimiento de los árboles, que se simula para los siguientes nueve años, de forma que la ejecución de claras permitirá mejorar el crecimiento diametral y, en consecuencia, la posible superficie de descorche del árbol (a igualdad de altura de descorche) y la intensidad del descorche aplicado también afectará (en positivo o negativo, según se haya aplicado una intensidad mayor o menor) al crecimiento en sección de los árboles, que, a su vez se traducirá en una variación en la producción individual y del conjunto de los árboles de la parcela.

Esta simulación a futuro permite obtener las mismas salidas que los resultados inmediatos, pero incorporando las variaciones simuladas y el crecimiento de los árboles remanentes de acuerdo con las mismas.

Hay que advertir aquí que el modelo de crecimiento empleado no está ajustado a las condiciones de crecimiento de Pino Gordo, pues no se trata de obtener un modelo de crecimiento y producción para estimar la producción real del monte sino una herramienta de simulación, suficientemente realista, que permita poner visiblemente de manifiesto los efectos de las actuaciones sobre las características resultantes de la masa, tanto en términos selvícolas como ecológicos y productivos. Con este fin se ha permitido que los crecimientos simulados sean bastante elevados, si se comparan con los valores de la literatura, con el fin de ayudar a amplificar la visualización de los efectos que se pretenden poner de manifiesto a través de la simulación.

\section{b) Aplicación práctica con alumnos}

Durante el curso en que se ha puesto en marcha el marteloscopio, se han realizado pruebas de aplicación con alumnos colaboradores, tanto del Grado de Ingeniería Forestal como del Máster en Ingeniería de Montes, que han colaborado en el establecimiento de la herramienta y en las pruebas de control para su correcto funcionamiento. También se ha hecho una presentación del marteloscopio en una clase práctica a todos los alumnos de la asignatura Selvicultura Mediterránea.

En cursos próximos se incorporará la realización de una práctica efectiva que tendrá carácter multidisciplinar, implicando a varias de las asignaturas de ambas titulaciones. Los alumnos propondrán el aprovechamiento de corcho a realizar, concretado en la selección de los árboles a descorchar y las alturas de descorche a emplear en cada árbol, así como los tratamientos selvícolas a realizar con el fin de mejorar las producciones futuras del monte. Estos datos, incorporados al simulador, se traducirán en unos valores económicos respecto al corcho a obtener en el aprovechamiento simulado y en los efectos inmediatos y a medio/largo plazo sobre los aprovechamientos y el valor productivo y ecológico del monte en el futuro. 
La simulación en tiempo real en el monte de actuaciones de aprovechamiento y de gestión selvícola permite a los alumnos jugar con la variación de su propuesta y realizar así un análisis de sensibilidad intuitivo ya que el grupo puede ir viendo y discutiendo, en tiempo real, los resultados de las actuaciones que va ejecutando en el simulador y, por tanto, corregir las mismas hasta alcanzar los objetivos de gestión que se le propongan en la actividad. Esto mejorará sin duda su aprendizaje y el conocimiento de las interacciones entre las decisiones tomadas para la ejecución de los aprovechamientos forestales y de la selvicultura y la evolución que la masa forestal tendrá como consecuencia. Y no sólo en relación con los objetivos económicos de la gestión, sino también con los aspectos ecológicos y la biodiversidad del sistema forestal.

\section{RESULTADOS}

Los resultados obtenidos en este Proyecto de Innovación Docente pueden resumirse en los siguientes:

Disponibilidad de una parcela permanente (Marteloscopio) en monte alcornocal, monitorizada y marcada sobre el terreno para su utilización en actividades formativas y de demostración de tratamientos selvícolas y de gestión del aprovechamiento de corcho. Se ha realizado con la colaboración de los dueños y gestores de la finca "Pino Gordo" donde se ha establecido el dispositivo, lo que permitirá mantener su integridad y facilitar su utilización durante un tiempo suficiente, sin modificaciones significativas de su composición y estructura para el fin educativo previsto, gracias al Convenio de Colaboración Educativa existente entre la Empresa PADEPAMA, S.L. gestora de la finca y la Universidad de Córdoba.

Uno de los resultados más interesantes del proyecto, junto con el establecimiento y monitorización del Marteloscopio en monte alcornocal, es el software desarrollado específicamente para su utilización. Dicho software es el que permite realizar la simulación de las intervenciones selvícolas y de aprovechamiento del corcho en el propio monte y obtener simultáneamente los resultados de las mismas en relación a sus efectos sobre el valor económico y ecológico de la masa forestal antes y después de las actuaciones simuladas, así como valorar el coste económico de los objetivos ecológicos que pueden subyacer en la conservación de árboles de elevado valor ecológico y escaso o nulo valor económico y que puedan afectar al valor económico global de la masa, y viceversa: estimar el coste ecológico de las actuaciones simuladas encaminadas a mejorar el estado productivo de la masa.

Como resultado del proyecto se ha presentado un Trabajo Fin de Grado por uno de los miembros del equipo sobre el proceso de diseño, instalación y puesta a punto del Marteloscopio.

A continuación, se presentan algunas salidas gráficas y numéricas del simulador obtenidas con la herramienta desarrollada: 




Figura 2. Mapas de árboles. El tamaño de los círculos es función del tamaño de los árboles (fila superior), de su valor ecológico (fila intermedia) y de la superficie de descorche (fila inferior). Los mapas de la columna izquierda representan el momento actual y los de la derecha al cabo de 9 años. Los círculos amarillos representan los árboles cortados en la simulación. 
Abellanas, B., Baldero, F., Guada, L., Cuadros, S., Pérez, P.J., Sellez, A. \& Urbano, E.

En la tabla 1 y Figura 3 se muestran los resultados, en el momento actual, de una simulación:

Tabla 1.

Resultados cuantitativos tras la simulación, en el momento actual: Valores dasométricos, ecológicos y productivos.

\begin{tabular}{|c|c|c|c|c|c|c|c|c|c|}
\hline \multicolumn{8}{|c|}{ Masa inicial } & & \\
\hline $\mathrm{N}$ (pies/ha) & $\mathrm{G}(\mathrm{m} 2 / \mathrm{ha})$ & $\mathrm{gm}(\mathrm{cm} 2)$ & $D_{-} m(\mathrm{~cm})$ & $\mathrm{HDm}(\mathrm{m})$ & $\mathrm{IDm}$ & SD total $(\mathrm{m} 2)$ & EcoVal_m & & \\
\hline 309.33 & 11.39 & 368.31 & 21.66 & 1.89 & 33.30 & 172.94 & 6.01 & & \\
\hline \multicolumn{8}{|c|}{ Masa extraída } & \multicolumn{2}{|c|}{ CORCHO EXTRAIDO } \\
\hline $\mathrm{N}$ (pies/ha) & $\mathrm{G}(\mathrm{m} 2 / \mathrm{ha})$ & $\mathrm{gm}(\mathrm{cm} 2)$ & D_m (cm) & $\mathrm{HDm}(\mathrm{m})$ & $\mathrm{IDm}$ & SD total $(\mathrm{m} 2)$ & EcoVal & SD_Born (m2) & SD_Rep (m2) \\
\hline 74.67 & 3.41 & 456.09 & 24.10 & 2.05 & 34.095 & 50.13 & 8.27 & 0.00 & 172.94 \\
\hline \multicolumn{8}{|c|}{ Masa remanente } & & \\
\hline $\mathrm{N}$ (pies/ha) & $\mathrm{G}(\mathrm{m} 2 / \mathrm{ha})$ & $\mathrm{gm}(\mathrm{cm} 2)$ & D_m (cm) & $\mathrm{HDm}(\mathrm{m})$ & $\mathrm{IDm}$ & SD total $(\mathrm{m} 2)$ & EcoVal & \multicolumn{2}{|c|}{ Variación del Valor Ecológico } \\
\hline 234.67 & 7.99 & 340.38 & 20.82 & 1.84 & 33.03 & 122.82 & 5.29 & \multicolumn{2}{|c|}{$-11.97 \%$} \\
\hline
\end{tabular}

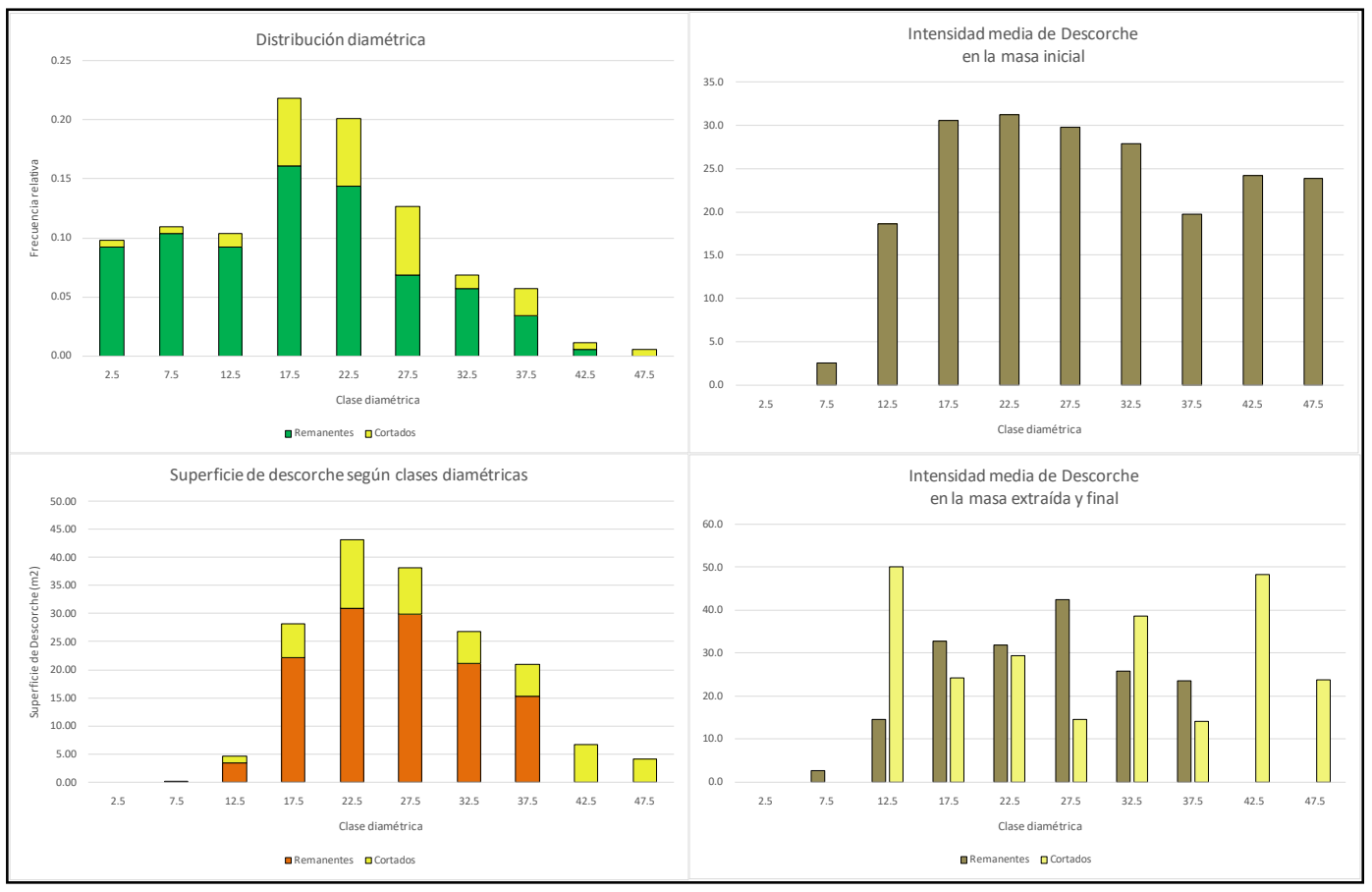

Figura 3. Resultados gráficos de la simulación en el momento actual: Histogramas de frecuencias de los valores dasométricos y productivos por clases diamétricas. En amarillo los árboles cortados en la simulación

En la tabla 2 y Figura 4 se muestran los resultados de la simulación realizada, al cabo de nueve años:

Tabla 2.

Resultados cuantitativos tras nueve años desde la simulación de la actuación.

\begin{tabular}{|c|c|c|c|c|c|c|c|c|}
\hline \multicolumn{7}{|c|}{ Masa tras 9 años } & \multirow[b]{2}{*}{ EcoVal_m } & \multirow[b]{2}{*}{ Aumento del valor ecológico en 9 años } \\
\hline $\mathrm{N}$ (pies/ha) & $G$ (m2/ha) & $\mathrm{gm}(\mathrm{cm} 2)$ & D_m $(\mathrm{cm})$ & $\mathrm{HDm}(\mathrm{m})$ & $\mathrm{IDm}$ & SD total $(\mathrm{m} 2)$ & & \\
\hline 234.67 & 14.20 & 605.20 & 27.76 & 1.89 & 23.97 & 165.77 & 5.55 & $4.97 \%$ \\
\hline
\end{tabular}




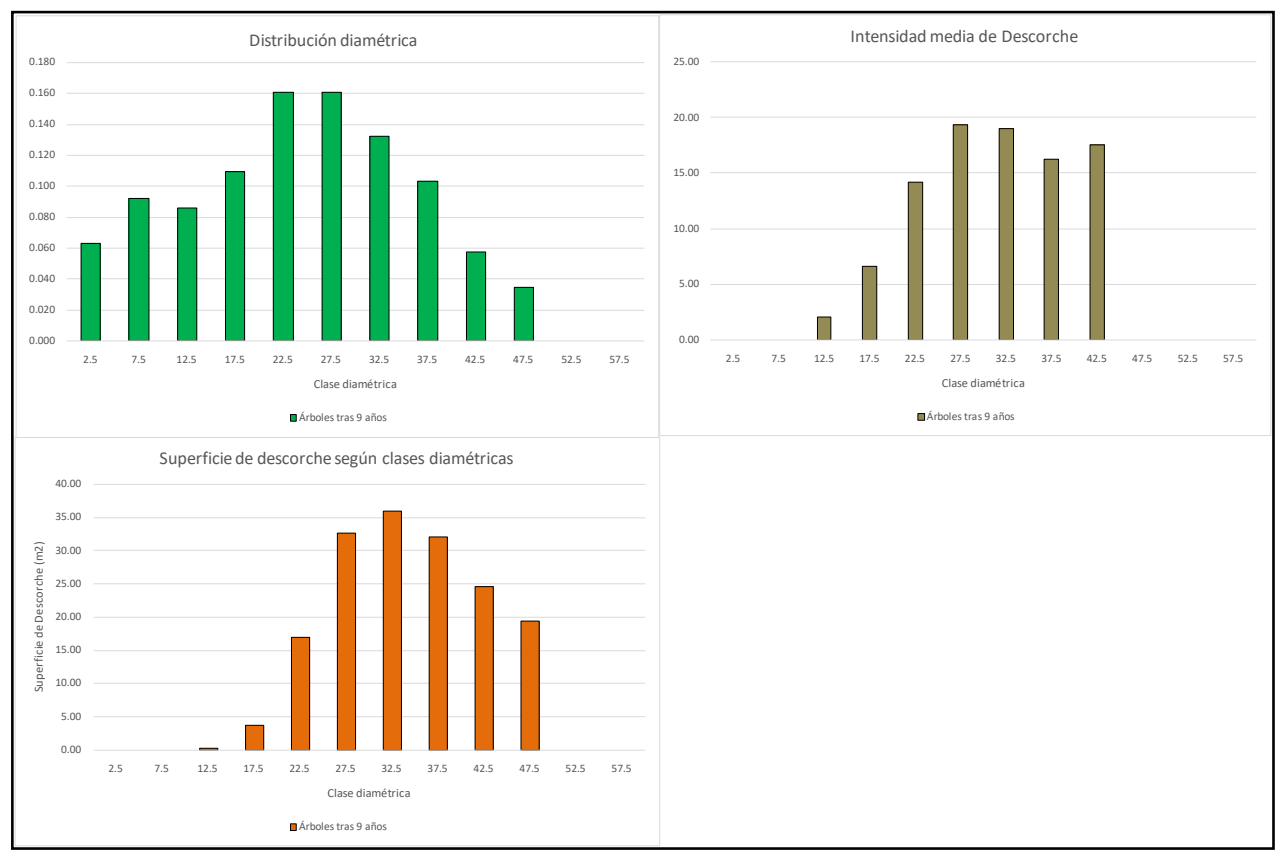

Figura 4. Resultados gráficos al cabo de nueve años de la simulación: Histogramas de frecuencias de los valores dasométricos y productivos por clases diamétricas.

\section{CONCLUSIONES}

Como principal conclusión del trabajo llevado a cabo se extrae la gran utilidad del dispositivo desarrollado para la enseñanza y el aprendizaje de la selvicultura y la gestión del aprovechamiento del corcho en condiciones reales de campo sin renunciar al análisis de los resultados de las actuaciones, tanto de forma inmediata a las actuaciones como sus efectos al cabo de un turno de descorche.

En este proyecto se ha podido aunar la adquisición de destrezas prácticas sobre el terreno en la toma de decisiones sobre el objeto último de la gestión forestal que es el bosque o masa forestal con el análisis cuantitativo y crítico de las decisiones que hasta ahora requerían una disociación de ambas etapas en el tiempo. La observación in situ de los resultados selvícolas y de la producción de corcho obtenidos a raíz de las decisiones adoptadas en monte, tanto de forma inmediata como al cabo del tiempo, permitirán, sin duda, adquirir una mejor comprensión de los objetivos y criterios a emplear en la gestión cuidadosa y eficiente del monte alcornocal a los alumnos de las titulaciones relacionadas con la gestión forestal.

La herramienta desarrollada en este proyecto contribuirá de forma decisiva a facilitar a los alumnos la adquisición de algunas de las competencias más importantes que deben adquirir los titulados en el Grado de Ingeniería Forestal en su formación tales como la capacidad de resolución de problemas con creatividad, iniciativa, metodología y razonamiento crítico, o la capacidad de trabajar en equipos multidisciplinares, entre las competencias básicas, y conocer, comprender y utilizar los principios de la Selvicultura y de los Aprovechamientos forestales entre las competencias Comunes de la Rama Forestal 


\section{Agradecimientos:}

A la Universidad de Córdoba por haber financiado este Proyecto de Innovación Docente a través del Plan de Innovación y Buenas Prácticas Docentes del curso 2017/2018.

A la empresa PADEPAMA S.L. por ceder la finca Pino Gordo para la instalación y posterior utilización docente del marteloscopio en monte alcornocal

A Luis Guada, miembro del equipo de trabajo, y la empresa INNOFOR por el apoyo técnico prestado en la utilización del equipo Field-Map.

\section{REFERENCIAS}

Abellanas, B., Cuadros, S., Pérez, P.J., \& Sellez, A. (2018) Diseño, Instalación y Aplicación de la herramienta Marteloscopio para el entrenamiento práctico y la incorporación efectiva de valores económicos, ecológicos y sociológicos a los tratamientos selvícolas en monte. Revista de Innovación y Buenas Prácticas Docentes UCO, 5, 80-84

Kraus, D., Bütler, R., Krumm, F., Lachat, T., Larrieu, L., Mergner, U., Paillet, Y., Rydkvist, T., Schuck, A., \& Winter, S. (2016). Catalogue of tree microhabitats Reference field list. Integrate+ Technical Paper. 16p.

Ribeiro, N.A., \& Surov'y, P. (2011). Growth Modeling in Complex Forest Systems: CORKFITS a Tree SpatialGrowth Model for Cork Oak Woodlands. Formath 10, 263-278. 